\title{
Scale Independent Workable Model of Final Unification
}

\author{
U. V.S. Seshavatharam ${ }^{1, *}$, S. Lakshminarayana ${ }^{2}$ \\ ${ }^{1}$ Honorary Faculty, I-SERVE, Survey no-42, Hitex road, Hitech city, Hyderabad-84, Telangana, India \\ ${ }^{2}$ Department of Nuclear Physics, Andhra University, Visakhapatnam-03, AP, India
}

Copyright $\bigcirc 2016$ by authors, all rights reserved. Authors agree that this article remains permanently open access under the terms of the Creative Commons Attribution License 4.0 International License

\begin{abstract}
We show that, Schwarzschild radius of Planck mass plays a vital role in electroweak and strong interactions. With reference to the observed large proportionality ratio, $0.1153\left(m_{p} / m_{e}\right)^{12}$, it seems appropriate to consider a large nuclear gravitational constant, $G_{s} \cong 3.3295608 \mathrm{~m}^{3} \mathrm{~kg}^{-1} \mathrm{sec}^{-2}$. Qualitatively this idea is in agreement with "Strong gravity" concept proposed by Abdus Salam and C.Sivaram [Mod. Phys. Lett., A8(4), 321- 326. (1993)]. We would like to suggest that, by replacing the Newtonian gravitational constant with the proposed nuclear gravitational constant, predicted high energy levels of String theory can be brought down to the current hadronic scale. Based on this idea, we defined the nuclear Planck mass, $m_{n p l} \cong \sqrt{\hbar c / G_{s}} \cong 546.7 \mathrm{MeV} / c^{2}$ and proposed a quantized model mechanism for understanding the hadronic mass spectrum.
\end{abstract}

Keywords Final Unification, Nuclear Gravitational Constant, Newtonian Gravitational Constant, String Theory, Nuclear Planck Mass, Neutral Charge Nuclear Dark Baryon, Quantized Hadronic Mass Spectrum

\section{Introduction}

A Grand Unified Theory (GUT) is a model in particle physics in which at high energy, the three gauge interactions of the Standard Model which define the electromagnetic, weak, and strong interactions, are merged into one single force. Unifying gravity with the other three interactions would provide a theory of everything (TOE) [1-4]. In general, GUT is often seen as an intermediate step towards a TOE. The most desirable cases of any unified description [4] are:

1) To simplify the complicated issues of known physics.

2) To predict new effects, arising from a combination of the fields inherent in the unified description.

So far it has never been achieved. In this paper, we geared up the following things.

A). By considering the Schwarzschild radius of Planck mass and proton-electron mass ratio, we proposed very simple relations among the Newtonian gravitational constant, Fermi's weak coupling constant, Strong coupling constant and nuclear charge radius.

B). With reference to the proposed semi empirical relations, it is possible to show that, proportionality ratio is of the order of $0.1153\left(m_{p} / m_{e}\right)^{12}$.

C). By replacing the large proportionality ratio with a large gravitational constant, $G_{s} \cong 3.3295608 \mathrm{~m}^{3} \mathrm{~kg}^{-1} \mathrm{sec}^{-2}$, assumed to be associated with nuclear structure, we eliminated the higher powers of proton-electron mass ratio and proposed unified and simplified semi empirical relations.

D). By replacing the Newtonian gravitational constant with the proposed nuclear gravitational constant, we defined the nuclear Planck mass, $m_{n p l} \cong \sqrt{\hbar c / G_{s}} \cong 546.7 \mathrm{MeV} / c^{2}$ and developed a quantized model mechanism for understanding the hadronic mass spectrum.

\section{Conceptual Thought Connected with Final Unification}

Conceptual thought: Schwarzschild radius of Planck mass plays a vital role in electroweak and strong interactions.

Let, $G_{N} \cong 6.67408 \times 10^{-11} \mathrm{~m}^{3} \mathrm{~kg}^{-1} \mathrm{sec}^{-2}$.

Planck mass $=M_{p l} \cong \sqrt{\frac{\hbar c}{G_{N}}} \cong 2.176471826 \times 10^{-8} \mathrm{~kg}$

$R_{\text {spl }} \cong$ Schwarzschild radius of Planck mass

$\cong \frac{2 G_{N} M_{p l}}{c^{2}} \cong 2 \sqrt{\frac{G_{N} \hbar}{c^{3}}} \cong 3.2324592 \times 10^{-35} \mathrm{~m}$

\section{Strange Result Connected with Planck Scale Schwarzschild Radius}

Let, $\left(\frac{m_{p}}{m_{e}}\right)$ be the proton-electron mass ratio and $G_{F}$ be 
the Fermi's weak coupling constant.

It is noticed that,

$$
\left(\frac{m_{p}}{m_{e}}\right) \cong\left(\frac{G_{F}}{\hbar c R_{s p l}^{2}}\right)^{\frac{1}{10}} \cong\left(\frac{G_{F} c^{2}}{4 G_{N} \hbar^{2}}\right)^{\frac{1}{10}}
$$

Based on this relation,

$$
\begin{aligned}
& G_{F} \cong\left\{\left(\frac{m_{p}}{m_{e}}\right)^{10}\right\}\left(\frac{4 G_{N} \hbar^{2}}{c^{2}}\right) \cong 1.438965 \times 10^{-62}{\mathrm{~J} . \mathrm{m}^{3}}^{3} \\
& G_{N} \cong\left(\frac{m_{e}}{m_{p}}\right)^{10}\left(\frac{G_{F} c^{2}}{4 \hbar^{2}}\right) \\
& \frac{G_{F}}{G_{N}} \cong\left\{\left(\frac{m_{p}}{m_{e}}\right)^{10}\right\}\left(\frac{4 \hbar^{2}}{c^{2}}\right)
\end{aligned}
$$

If, recommended $G_{F} \cong 1.435850984 \times 10^{-62}{\mathrm{~J} . \mathrm{m}^{3}}^{3}$, obtained $G_{N} \cong 6.65963739 \times 10^{-11} \mathrm{~m}^{3} \mathrm{~kg}^{-1} \mathrm{sec}^{-2}$

\section{Strange Result Connected with Fermi's Weak Coupling Constant}

Let, $R_{0} \approx 1.24 \mathrm{fm}$ be the nuclear charge radius.

It is noticed that,

$$
\left(\frac{m_{p}}{m_{e}}\right) \cong \sqrt{\frac{\hbar c R_{0}^{2}}{G_{F}}}
$$

From relations (1) and (5),

$$
\begin{gathered}
R_{0} \cong\left(\frac{m_{p}}{m_{e}}\right)^{6} \sqrt{\frac{4 \hbar G_{N}}{c^{3}}} \cong\left(\frac{m_{p}}{m_{e}}\right)^{6} R_{s p l} \\
\frac{R_{0}}{R_{s p l}} \cong\left(\frac{m_{p}}{m_{e}}\right)^{6} \quad \text { (Or) } \frac{\pi R_{0}^{2}}{\pi R_{s p l}^{2}} \cong\left(\frac{m_{p}}{m_{e}}\right)^{12}
\end{gathered}
$$

If, $\quad G_{N} \cong 6.67408 \times 10^{-11} \mathrm{~m}^{3} \mathrm{~kg}^{-1} \mathrm{sec}^{-2}$, obtained $R_{0} \cong 1.238755 \mathrm{fm}$.

\section{Strange Result Connected with Strong Coupling Constant}

Let, $\alpha_{s} \cong 0.1153$ be the strong coupling constant.

It is noticed that,

$$
\left(\frac{m_{p}}{m_{e}}\right)^{12} \cong\left(\frac{1}{\alpha_{s}}\right)\left(\frac{\hbar c}{G_{N} m_{p}^{2}}\right)
$$

From relations (6) and (7)

$$
\begin{aligned}
\alpha_{s} & \cong\left(\frac{m_{e}}{m_{p}}\right)^{12}\left(\frac{\hbar c}{G_{N} m_{p}^{2}}\right) \cong\left(\frac{R_{s p l}}{R_{0}}\right)^{2}\left(\frac{\hbar c}{G_{N} m_{p}^{2}}\right) \\
& \cong \frac{M_{p l}^{2} R_{s p l}^{2}}{m_{p}^{2} R_{0}^{2}} \cong\left(\frac{M_{p l} R_{s p l} c}{m_{p} R_{0} c}\right)^{2} \cong\left(\frac{2 \hbar}{m_{p} R_{0} c}\right)^{2}
\end{aligned}
$$

From above relations, we would like to say that, magnitude of $\alpha_{s}$ seems to be around 0.1153. The same conclusion can also be extracted from Particle data group's (PDG) review on Quantum chromodynamics [5]. See the following table-1.

\section{To Eliminate the Higher Powers of Proton-electron Mass Ratio}

It is true that, unless stringent requirements are met, in general, speculative alternatives to currently accepted theories cannot be accepted or published. Scientific papers having content that lie outside the mainstream of current

\begin{tabular}{|c|c|c|c|}
\hline 1 & $\alpha_{s}\left(M_{Z}^{2}\right)=0.1161_{-0.0048}^{+0.0041}$ & 7 & $\alpha_{s}\left(M_{Z}^{2}\right)=0.1158 \pm 0.0035$ \\
\hline 2 & $\alpha_{s}\left(M_{Z}^{2}\right)=0.1151_{-0.0087}^{+0.0093}$ & 8 & $\alpha_{s}\left(M_{Z}^{2}\right)=0.1154 \pm 0.0020$ \\
\hline 3 & $\begin{aligned} \alpha_{s}\left(M_{Z}^{2}\right) & =0.1148 \pm 0.0014(\exp .) \\
& \pm 0.0018(P D F)_{-0.0000}^{+0.0050}\end{aligned}$ & 9 & $\alpha_{s}\left(M_{Z}^{2}\right)=0.1131_{-0.0022}^{+0.0028}$ \\
\hline 4 & $\alpha_{s}\left(M_{Z}^{2}\right)=0.1134 \pm 0.0011$ & 10 & $\alpha_{s}\left(M_{Z}^{2}\right) \cong 0.1156_{-0.0022}^{+0.0021}$ \\
\hline 5 & $\alpha_{s}\left(M_{Z}^{2}\right)=0.1142 \pm 0.0023$ & 11 & $\alpha_{s}\left(M_{Z}^{2}\right) \cong 0.1156_{-0.0034}^{+0.0041}$ \\
\hline 6 & $\alpha_{s}\left(M_{Z}^{2}\right)=0.1151_{-0.0032}^{+0.0033}$ & 12 & $\alpha_{s}\left(M_{Z}^{2}\right) \cong 0.1151_{-0.0087}^{+0.0093}$ \\
\hline
\end{tabular}
research must justify by including a clear, detailed discussion of the motivation for the new speculation, with reasons for introducing any new concepts. If the new formulation results are in contradiction with the accepted theory, then there must both be a discussion of which experiments could be done to verify that the conventional theory needs improvement, and also an analysis showing the consistency of the new theory with the existing experiments.

Table 1. Magnitude of $\alpha_{s}$ close to 0.1153 
In this context, by considering a proportionality ratio of $0.1153\left(m_{p} / m_{e}\right)^{12}$, we propose the following workable assumption: In nuclear structure, there exists a very large gravitational constant, $G_{s} \cong 3.3295608 \mathrm{~m}^{3} \mathrm{~kg}^{-1} \mathrm{sec}^{-2}$. We estimated this magnitude by the following ad-hoc relation.

$$
\begin{gathered}
\text { Planck's constant, } h \cong \sqrt{\left(\frac{m_{p}}{m_{e}}\right)\left(\frac{e^{2}}{4 \pi \varepsilon_{0} c}\right)\left(\frac{G_{s} m_{p}^{2}}{c}\right)} \\
\rightarrow G_{s} \cong\left(\frac{4 \pi \varepsilon_{0} h^{2} c^{2} m_{e}}{e^{2} m_{p}^{3}}\right)
\end{gathered}
$$

Qualitatively, this assumption is not new and is having a long standing history [6-14]. For more information, readers are encouraged to see Abdus Salam's 'Strong gravity' concept [12]. In the early seventies Abdus Salam and his co-workers proposed the concept of strong gravity. In this context, in physics literature one can see valuable papers on 'strong gravity' proposed by C. J. Isham, Abdus Salam, C. Sivaram, J. Strathdee, K. P. Sinha, Y. Ne'eman and Dj. Sijacki. In Strong gravity, the successive self-interaction of a nonlinear spin-2 field was used to describe a non-abelian field of strong interactions. This idea was formulated in a two-tensor theory of strong and gravitational interactions, where the strong tensor fields are governed by Einstein-type field equations with a strong gravitational constant $G_{f} \approx 10^{38}$ times the Newtonian gravitational constant, $G_{N}$. Within the framework of this proposal, tensor fields were identified to play a fundamental role in the strong-interaction physics of quantum chromodynamics (QCD). Modifying these concepts, O. F. Akinto and Farida Tahir recently posted their work in arXiv preprint [14]. They elaborately discussed on modified strong gravity concepts pertaining to QCD and general relativity. In 2013. Roberto Onofrio [15] proposed a very interesting concept: Weak interactions are peculiar manifestations of quantum gravity at the Fermi scale, and that the Fermi coupling constant is related to the Newtonian constant of gravitation. In his opinion, at atto-meter scale, Newtonian gravitational constant seems to reach a magnitude of $8.205 \times 10^{22} \mathrm{~m}^{3} \mathrm{~kg}^{-1} \mathrm{sec}^{-2}$.

With this assumption, $10^{\text {th }}$ and $12^{\text {th }}$ powers of $\left(m_{p} / m_{e}\right)$ can be eliminated. In our recent published papers [16-23] and conference proceedings [24-28], we proposed that, there exist two large pseudo gravitational constants associated with nuclear and electromagnetic interactions and presented many interesting applications starting from nuclear radii to neutron star radius. By eliminating the two pseudo gravitational constants, in this paper, we proposed the above relations (1) to (9). See the following simplified relations (11) to (24).

\section{Application-1}

Strong coupling constant,

$$
\alpha_{s} \cong\left(\frac{2 \hbar}{m_{p} R_{0} c}\right)^{2} \cong\left(\frac{\hbar c}{G_{s} m_{p}^{2}}\right)^{2} \cong 0.1153
$$

\section{Application-2}

Proton rest mass, $m_{p} \cong\left[\left(\frac{G_{s}}{G_{N}}\right)\left(\frac{G_{s} m_{e}^{2}}{\hbar c}\right)\right]^{\frac{1}{10}} m_{e} \cong\left[\frac{G_{s} m_{e}^{2}}{G_{N} m_{n p l}^{2}}\right]^{\frac{1}{10}} m_{e}$ where $\sqrt{\frac{\hbar c}{G_{s}}} \cong 546.7 \mathrm{MeV} / c^{2} \cong$ Nuclear Planck mass $\cong m_{n p l}$

\section{Application-3}

Nuclear charge radius,

$$
R_{0} \cong \frac{2 G_{s} m_{p}}{c^{2}} \cong 1.238755 \mathrm{fm}
$$

\section{Application-4}

Root mean square radius of proton,

$$
R_{p} \cong \frac{\sqrt{2} G_{s} m_{p}}{c^{2}} \cong 0.875932 \mathrm{fm}
$$

\section{Application-5}

Fermi's Weak coupling constant,

$$
G_{F} \cong \hbar c\left(\frac{2 G_{s} m_{e}}{c^{4}}\right)^{2} \cong \frac{4 G_{s}^{2} m_{e}^{2} \hbar}{c^{3}} \cong G_{s} m_{e}^{2}\left(\frac{4 G_{s} \hbar}{c^{3}}\right)
$$

where $\sqrt{\frac{4 G_{s} \hbar}{c^{3}}} \cong \frac{2 G_{s} m_{n p l}}{c^{2}} \cong 0.36 \mathrm{fm} \cong$ Nuclear Planck length.

\section{Application-6}

Stable mass number,

$$
A_{s} \cong 2 Z+\left\{\left(\frac{G_{s} m_{p} m_{e}}{\hbar c}\right)(2 Z)\right\}^{2} \cong 2 Z+0.00642(Z)^{2}
$$

\section{Application-7}

For $Z \geq 30$, close to stable atomic nuclides, nuclear binding energy [18],

$$
(B E)_{A s} \cong-(Z) \sqrt{\left(\frac{3}{5} \frac{e^{2}}{4 \pi \varepsilon_{0} R_{p}}\right)\left(\frac{3}{5} \frac{G_{s} m_{p}^{2}}{R_{p}}\right)} \cong-Z \times 19.8 \mathrm{MeV}
$$

\section{Application-8}

Neutron weak decay,

$$
\left(m_{n}-m_{p}\right) c^{2} \times t_{n} \approx\left(N_{A}\right)\left(\frac{G_{s} m_{p}^{2}}{c}\right)
$$


where, $\left(\frac{\sqrt{M_{p l} m_{e}}}{m_{p}}\right)^{3} \cong 5.9658075 \times 10^{23} \cong N_{A}$ is very close to Avogadro number.

\section{Application-9}

Weak coupling angle and up-down quark mass ratio,

$$
\begin{aligned}
& \sin \theta_{W} \cong \sqrt{\frac{4 \pi \varepsilon_{0} G_{s} m_{p} m_{e}}{e^{2}}} \cong\left(\frac{m_{u}}{m_{d}}\right) \cong 0.46893 \\
& \sin ^{2} \theta_{W} \cong \frac{4 \pi \varepsilon_{0} G_{s} m_{p} m_{e}}{e^{2}} \cong\left(\frac{m_{u}}{m_{d}}\right)^{2} \cong 0.219895
\end{aligned}
$$

where $m_{u}, m_{d}$ represent up and down quark masses respectively.

\section{Application-10}

Magnetic dipole moment of proton,

$\mu_{\text {proton }} \cong\left(\frac{m_{u}}{m_{d}}\right)\left(\frac{e G_{s} m_{p}}{c}\right) \cong 1.3957 \times 10^{-26} \mathrm{~J} /$ T esla

Magnetic dipole moment of neutron,

$$
\mu_{\text {neutron }} \cong\left(\frac{m_{u}}{m_{d}}\right)^{\frac{3}{2}}\left(\frac{e G_{s} m_{n}}{c}\right) \cong 9.57 \times 10^{-27} \mathrm{~J} / \mathrm{T} \text { esla }
$$

\section{Application-11}

A). If $\left(M_{N S}, m_{n}\right)$ represent the masses of neutron star and neutron, then,

$$
\frac{G_{N} M_{N S} m_{n}}{\hbar c} \approx \sqrt{\frac{G_{s}}{G_{N}}} .
$$

B). If $R_{N S}$ represents the neutron star radius, then,

$$
\frac{R_{N S}}{\left(\sqrt{G_{s} \hbar / c^{3}}\right)} \approx \sqrt{\frac{G_{s}}{G_{N}}}
$$

\section{Application-12}

Planet's earth's magnetic moment,

$$
\mu_{\text {earth }} \cong\left(\frac{\mu_{\text {proton }}}{\mu_{\text {electron }}}\right) *\left(\frac{e G_{s} M_{\text {earth }}}{2 c}\right) \cong 8.15 \times 10^{22} \mathrm{~J} . T e s l a^{-1} \text {. }
$$

\section{Application-13}

From relations (10) and (12), Newtonian gravitational constant,

$$
\begin{aligned}
& G_{N} \cong\left(\frac{m_{e}}{m_{p}}\right)^{12}\left(\frac{G_{s}^{2} m_{p}^{2}}{\hbar c}\right) \cong\left(\frac{h}{\hbar}\right)\left(\frac{m_{e}}{m_{p}}\right)^{14}\left(\frac{4 \pi \varepsilon_{0} h c}{e^{2}}\right)^{2}\left(\frac{h c}{m_{p}^{2}}\right) \\
& \cong 2 \pi\left(\frac{m_{e}}{m_{p}}\right)^{14}\left(\frac{4 \pi \varepsilon_{0} h c}{e^{2}}\right)^{2}\left(\frac{h c}{m_{p}^{2}}\right) \cong 6.679856051 \times 10^{-11} \mathrm{~m}^{3} \mathrm{~kg}^{-1} \mathrm{sec}^{-2}
\end{aligned}
$$

All these relations clearly indicate and establish the role of the proposed large nuclear gravitational constant. It may be noted that.

1) Relation (11) is a very simple relation pertaining to strong coupling constant.

2) Relation (12) indicates the massive origin of proton and needs special attention.

3) Relation (13) is a direct consequence of relation (11).

4) Relation (14) is a best fit for the recommended value of the root mean square radius of proton.

5) Relation (15) is a best fit in electroweak sector.

6) Relations (16) and (17) clearly indicate the simplified versions of nuclear stability and binding energy.

7) Relation (18) seems to be a good clue in understanding the weak decay of neutron.

8) Relations (19) and (20) seem to be a nice fit for understanding weak coupling angle, up and down quark masses and magnetic dipole moments of nucleons.

9) Relations (21) and (22) clearly indicate the best possible astrophysical applications.

10) Relation (23) is a nice fit for understanding planetary and exo-planetary 'mass dependent' magnetic dipole moments.

11) Relation (24) indicates a possible relation in between the micro-macro elementary physical constants.

Based on these points, we would like to stress that,

1) In the development of science and engineering, 'data fitting' and 'workability' are the two essential tools by using which physical models can be generated and validated in a progressive manner.

2) The problem is with 'our understanding and our perception' by using which the current 'scientific standards' and 'procedures' can be reviewed for a better understanding of nature.

3) With reference to String theory models and Quantum gravity models, proposed nuclear gravitational constant and presented results can be given some consideration in developing a 'workable model' of 'scale independent final unification'.

4) Considering the wide applicable range of the proposed assumption, we are confident to say that, with further research and analysis, 'hidden and left over physics' can easily be explored.

\section{To Synchronize Our Model with String Theory and Electroweak Theory}

The basic idea of String theory is very simple, very interesting and highly intuitive. Hence most of the science community strongly believes in String theory and its super child 'M-theory'. Unfortunately, there are no concrete new predictions of string theories on low energy scales and high energy scale predictions are beyond the reach of current technology. In addition, with string theory, so far, no one could estimate/implement the Newtonian gravitational 
constant in nuclear interactions. Many critics of string theory have expressed concerns about the large number of possible solutions and possible universes described by string theory. To sustain the ideology of String theory, to keep its mathematical beauty intact and to make it as a practical model of the nucleus, we would like to emphasize the following two points.

1) String theory was originally introduced for understanding the basic hadronic mass spectrum.

2) By replacing the Newtonian gravitational constant with the proposed strong interaction gravitational constant, original energy levels of String theory can be brought down to the current LHC scale.

Electroweak theory is very important for modern cosmology, particularly on how the universe evolved. This is because shortly after the Big Bang, the temperature was approximately above $10^{15} \mathrm{~K}$. Electromagnetic force and weak force were merged into a combined electroweak force. Very interesting feature is that, in our model, we proposed useful relations for fitting and understanding the Fermi's electroweak coupling constant, weak coupling angle and neutron's weak decay. Proceeding further, we proposed a very interesting relation for understanding the proton magnetic moment. Extending this relation, we made another bold attempt to fit and understand our earth's magnetic dipole moment.

\section{To Understand the Hadronic Mass Spectrum}

In 2010, we published our paper on super symmetry with title "Supersymmetry in strong and weak interactions". Interested readers may refer [29-34]. The basic concepts were:

1) Fermion - boson mass ratio is very close 2.26.

2) Integral charge quarks exist in bosonic form.

3) Estimated rest energies of up, down and strange quark bosons are, $1.94 \mathrm{MeV}, 4.2 \mathrm{MeV}$ and $67.4 \mathrm{MeV}$ respectively.

4) Neutral pion is nothing but the pair of strange quark bosons. Charged pion constitutes neutral pion and down quark boson.

5) Being lighter in mass, up, down and strange quark bosons combine with heavy neutral baryons and generate charged baryons.

In this section, we propose a model mechanism for understanding the hadronic mass spectrum having a neutral charge. Basic idea is that, $\left(\frac{\hbar c}{G_{s}}\right)^{1 / 2} \cong 546.7 \mathrm{MeV} / c^{2}$ can be called as the Nuclear Planck Mass having a neutral charge It can also be called as the 'neutral charge nuclear dark baryon' [9]. Basic hadronic mass spectrum can be understood with the following relation. $\left(m_{\text {baryon }} c^{2}\right)_{n} \cong(n)^{\frac{1}{4}}\left(\frac{1}{\alpha_{s}}\right)^{\frac{1}{4}} \sqrt{\frac{\hbar c^{5}}{G_{s}}} \cong(n)^{\frac{1}{4}}\left(\frac{1}{\alpha_{s}}\right)^{\frac{1}{4}} 546.7 \mathrm{MeV}$

where $\alpha_{\mathrm{s}} \cong$ Strong coupling constant $\cong 0.1152$

and $n=1,2,3,4, \ldots$

First generation neutral baryons can be classified with relation (25). See the following table-2. Sub levels can be approximated with further study and analysis.

Table 2. To fit the basic baryonic mass spectrum

\begin{tabular}{|c|c|c|c|}
\hline$n$ & $\left(m_{\text {baryon }} c^{2}\right)_{n}$ & $n$ & $\left(m_{\text {baryon }} c^{2}\right)_{n} \mathrm{MeV}$ \\
\hline 1 & 938.4 & 7 & 1526.4 \\
\hline 2 & 1116.0 & 8 & 1578.2 \\
\hline 3 & 1235.0 & 9 & 1625.4 \\
\hline 4 & 1327.1 & 10 & 1668.7 \\
\hline 5 & 1403.2 & 11 & 1709.0 \\
\hline 6 & 1468.7 & 12 & 1746.6 \\
\hline
\end{tabular}

Currently believed Lamda, Delta and Xi baryons seem to fall under first generation baryons. Based on integral charge quark bosons, sigma baryons can be considered as a combination of first generation baryon and up or down or strange quark boson.

For example,

$2^{\text {nd }}$ level neutral baryon, $1116.0 \mathrm{MeV}$ combines with strange quark boson of rest energy $67.4 \mathrm{MeV}$ and generates a charged baryon of rest energy $1183.4 \mathrm{MeV}$.

$4^{\text {th }}$ level neutral baryon, $1327.1 \mathrm{MeV}$ combines with strange quark boson of rest energy $67.4 \mathrm{MeV}$ and generates a charged baryon of rest energy 1394.5.4 MeV.

In some cases, either a neutral pion or a charged pion also combines with estimated neutral baryon and generates a neutral/charged baryon. The same idea can be applied to second and third generation neutral baryons.

Second generation neutral baryons can be estimated with,

$$
\left(m_{\text {baryon }} c^{2}\right)_{n} \cong(n)^{\frac{1}{4}}\left(\frac{1}{\alpha_{s}}\right)^{\frac{1}{2}} 546.7 \mathrm{MeV}
$$

See the following table- 3 .

Table 3. To fit the second generation baryonic mass spectrum

\begin{tabular}{|c|c|c|c|}
\hline$n$ & $\left(m_{\mathrm{MeV}} m_{\text {baryon }} c^{2}\right)_{n}$ & $n$ & $\left(m_{\mathrm{MeV}}{ }_{\text {baryon }} c^{2}\right)_{n}$ \\
\hline 1 & 1610.7 & 7 & 2620.0 \\
\hline 2 & 1915.5 & 8 & 2708.9 \\
\hline 3 & 2119.8 & 9 & 2789.9 \\
\hline 4 & 2277.9 & 10 & 2864.3 \\
\hline 5 & 2408.6 & 11 & 2933.4 \\
\hline 6 & 2520.9 & 12 & 2997.9 \\
\hline
\end{tabular}


Charmed and charmed strange baryons seem to fall under second generation baryons. It is very interesting to note that, 1) Ground state baryon of rest energy 1610.7 MeV seems to combine with strange quark boson of rest energy 67.4 MeV and generates a baryon of rest energy 1678.1 $\mathrm{MeV}$. With respect to mass, this can be compared with currently believed Omega baryon.

2) $2^{\text {nd }}$ level neutral baryon, $1915.5 \mathrm{MeV}$ combines with strange quark boson of rest energy $67.4 \mathrm{MeV}$ and generates a charged baryon of rest energy $1982.9 \mathrm{MeV}$.

Third generation neutral baryons can be estimated with,

$$
\left(m_{\text {baryon }} c^{2}\right)_{n} \cong(n)^{\frac{1}{4}}\left(\frac{1}{\alpha_{s}}\right) 546.7 \mathrm{MeV}
$$

See the following table-4.

Table 4. To fit the third generation baryonic mass spectrum

\begin{tabular}{|c|c|c|c|}
\hline$n$ & $\begin{array}{c}\left(m_{\text {baryon }} c^{2}\right)_{n} \\
\mathrm{MeV}\end{array}$ & $n$ & $\left(m_{\text {baryon }} c^{2}\right)_{n} \mathrm{MeV}$ \\
\hline 1 & 4745.7 & 7 & 7719.2 \\
\hline 2 & 5643.6 & 8 & 7981.2 \\
\hline 3 & 6245.6 & 9 & 8219.7 \\
\hline 4 & 6711.4 & 10 & 8439.1 \\
\hline 5 & 7096.4 & 11 & 8642.6 \\
\hline 6 & 7427.4 & 12 & 8832.7 \\
\hline
\end{tabular}

Bottom baryons seem to fall under third generation baryons. Interesting points to be are :

1) Rest energy of ground state baryon of third generation is $4745.7 \mathrm{MeV}$ and its corresponding meson rest energy can be considered as $9490 \mathrm{MeV}$ and can be compared with the first bottom-anti bottom rest energy of 9460 $\mathrm{MeV}$.

2) Second excited level of $4745.7 \mathrm{MeV}$ is $5643.6 \mathrm{MeV}$. It combines with up, down and strange bosons and generates charged baryons of rest energy $5645.6 \mathrm{MeV}$, $5647.8 \mathrm{MeV}$ and $5711 \mathrm{MeV}$. In this way, to some extent charged baryonic mass spectrum can be understood.

First generation neutral mesons can be expressed with, $\left(m_{\text {meson }} c^{2}\right)_{n} \cong\left[(n)^{\frac{1}{4}}\left(\frac{1}{\alpha_{s}}\right)^{\frac{1}{4}} 546.7 \mathrm{MeV}\right]+546.7 \mathrm{MeV}$

See the following table-5.
Table 5. To fit the first generation meson mass spectrum

\begin{tabular}{|c|c|c|c|}
\hline$n$ & $\underset{\mathrm{MeV}}{\left(m_{\text {meson }} c^{2}\right)_{n}}$ & $n$ & $\left(m_{\text {meson }} c^{2}\right)_{n}$ \\
\hline 1 & 1485.1 & 16 & 2423.5 \\
\hline 2 & 1662.7 & 17 & 2452.2 \\
\hline 3 & 1781.7 & 18 & 2479.6 \\
\hline 4 & 1873.8 & 19 & 2505.9 \\
\hline 5 & 1949.9 & 20 & 2531.2 \\
\hline 6 & 2015.4 & 21 & 2555.5 \\
\hline 7 & 2073.1 & 22 & 2579.0 \\
\hline 8 & 2124.9 & 23 & 2601.7 \\
\hline 9 & 2172.1 & 24 & 2623.7 \\
\hline 10 & 2215.4 & 25 & 2645.0 \\
\hline 11 & 2255.7 & 26 & 2665.7 \\
\hline 12 & 2293.3 & 27 & 2685.8 \\
\hline 13 & 2328.6 & 28 & 2705.3 \\
\hline 14 & 2361.9 & 29 & 2724.3 \\
\hline 15 & 2393.5 & 30 & 2742.9 \\
\hline
\end{tabular}

Currently believed and newly reported charmed and charmed strange mesons $[35,36]$ seem to fall under first generation mesons.

Second generation neutral mesons can be expressed with,

$$
\left(m_{\text {meson }} c^{2}\right)_{n} \cong\left[(n)^{\frac{1}{4}}\left(\frac{1}{\alpha_{s}}\right)^{\frac{1}{2}} 546.7 \mathrm{MeV}\right]+546.7 \mathrm{MeV}
$$

See the following table-6.

\begin{tabular}{|c|c|c|c|}
\hline$n$ & $\left(m_{\text {meson }} c^{2}\right)_{n} \mathrm{MeV}$ & $n$ & $\left(m_{\text {meson }} c^{2}\right)_{n} \mathrm{MeV}$ \\
\hline 1 & 2157.4 & 16 & 3768.2 \\
\hline 2 & 2462.2 & 17 & 3817.4 \\
\hline 3 & 2666.5 & 18 & 3864.4 \\
\hline 4 & 2824.6 & 19 & 3909.6 \\
\hline 5 & 2955.3 & 20 & 3953.0 \\
\hline 6 & 3067.6 & 21 & 3994.8 \\
\hline 7 & 3166.7 & 22 & 4035.1 \\
\hline 8 & 3255.6 & 23 & 4074.1 \\
\hline 9 & 3336.6 & 24 & 4111.8 \\
\hline 10 & 3411.0 & 25 & 4148.4 \\
\hline 11 & 3480.1 & 26 & 4183.9 \\
\hline 12 & 3544.6 & 27 & 4218.4 \\
\hline 13 & 3605.2 & 28 & 4251.9 \\
\hline 14 & 3662.4 & 29 & 4284.6 \\
\hline 15 & 3716.6 & 30 & 4316.4 \\
\hline
\end{tabular}

Table 6. To fit the second generation meson mass spectrum

Currently believed charmed and anti-charmed mesons and newly discovered and reported exotic mesons seem to fall under second generation mesons. 
Third generation neutral mesons can be expressed with, $\left(m_{\text {meson }} c^{2}\right)_{n} \cong\left[(n)^{\frac{1}{4}}\left(\frac{1}{\alpha_{s}}\right) 546.7 \mathrm{MeV}\right]+546.7 \mathrm{MeV}$

See the following table-7.

Table 7. To fit the second generation meson mass spectrum

\begin{tabular}{|c|c|c|c|}
\hline$n$ & $\underset{\mathrm{MeV}}{\left(m_{\text {meson }} c^{2}\right)_{n}}$ & $n$ & $\underbrace{}_{\mathrm{MeV}}\left(m_{\text {meson }} c^{2}\right)_{n}$ \\
\hline 1 & 5292.4 & 11 & 9189.3 \\
\hline 2 & 6190.3 & 12 & 9379.4 \\
\hline 3 & 6792.3 & 13 & 9557.9 \\
\hline 4 & 7258.1 & 14 & 9726.4 \\
\hline 5 & 7643.1 & 15 & 9886.1 \\
\hline 6 & 7974.1 & 16 & 10038.0 \\
\hline 7 & 8265.9 & 17 & 10183.0 \\
\hline 8 & 8527.9 & 18 & 10321.7 \\
\hline 9 & 8766.4 & 19 & 10454.7 \\
\hline 10 & 8985.8 & 20 & 10582.5 \\
\hline
\end{tabular}

Currently believed most of the bottom mesons, bottom and anti-bottom mesons of rest energy greater than $9490 \mathrm{MeV}$ seem to fall under third generation mesons.

By considering the excited levels of $546.7 \mathrm{MeV}$ as 1

$l^{\frac{1}{4}} \times 546.7 \mathrm{MeV}$ where $l=1,2,3, .$. sublevels of all the above mesons can be understood to some extent. For example, 4745.7 MeV baryon can be assumed to couple with $2^{\frac{1}{4}} \times 546.7 \mathrm{MeV}, 3^{\frac{1}{4}} \times 546.7 \mathrm{MeV}$, and $4^{\frac{1}{4}} \times 546.7 \mathrm{MeV}$ etc. and generates many sub level mesons. See the following table 8 .

Table 8. To understand the sub levels of $5292.4 \mathrm{MeV}$

\begin{tabular}{|c|c|c|c|}
\hline$l$ & $\begin{array}{c}\left(m_{\text {meson }} c^{2}\right)_{(n, l)} \\
\mathrm{MeV}\end{array}$ & $l$ & $\left(m_{m s o n} c^{2}\right)_{(n, l)} \mathrm{MeV}$ \\
\hline 1 & 5292.4 & 7 & 5634.949 \\
\hline 2 & 5395.84 & 8 & 5665.136 \\
\hline 3 & 5465.198 & 9 & 5692.612 \\
\hline 4 & 5518.851 & 10 & 5717.885 \\
\hline 5 & 5563.207 & 11 & 5741.328 \\
\hline 6 & 5601.332 & 12 & 5763.223 \\
\hline
\end{tabular}

Currently believed strange and other light mesons can be understood with the following two relations.

\section{First relation:}

$\left(m_{\text {meson }} c^{2}\right)_{n} \cong(n(n+1))^{\frac{1}{4}}\left[\frac{938.4 \mathrm{MeV}}{2.26}\right] \cong(n(n+1))^{\frac{1}{4}}[415.2 \mathrm{MeV}]$

where factor 2.26 is an ad hoc factor assumed to be connected with fermion-boson mass ratio pertaining to Super symmetry. It needs in depth analysis at fundamental level. See the following table-9.

Table 9. To fit light strange meson mass spectrum with $(n(n+1))^{1 / 4}$ levels

\begin{tabular}{|c|c|c|c|}
\hline$n$ & $\left(m_{\text {meson }} c^{2}\right)_{n} \mathrm{MeV}$ & $n$ & $\underset{\mathrm{MeV}}{\left(m_{\text {meson }} c^{2}\right)_{n}}$ \\
\hline 1 & 493.8 & 7 & 1135.8 \\
\hline 2 & 649.8 & 8 & 1209.5 \\
\hline 3 & 772.8 & 9 & 1278.8 \\
\hline 4 & 878.0 & 10 & 1344.6 \\
\hline 5 & 971.7 & 11 & 1407.3 \\
\hline 6 & 1057.0 & 12 & 1467.4 \\
\hline
\end{tabular}

\section{Second relation:}

$$
\left(m_{\text {meson }} c^{2}\right)_{n} \cong\left(\frac{n(n+1)}{2}\right)^{\frac{1}{4}}[415.2 \mathrm{MeV}]
$$

See the following table- 10 .

Table 10. To fit light and strange meson mass spectrum with $\left(\frac{n(n+1)}{2}\right)^{1 / 4}$ levels

\begin{tabular}{|c|c|c|c|}
\hline$n$ & $\left(m_{\text {meson }} c^{2}\right)_{n} \mathrm{MeV}$ & $n$ & $\begin{array}{c}\left(m_{m s o n} c^{2}\right)_{n} \\
\mathrm{MeV}\end{array}$ \\
\hline 1 & 415.2 & 7 & 955.1 \\
\hline 2 & 546.4 & 8 & 1017.0 \\
\hline 3 & 649.8 & 9 & 1075.4 \\
\hline 4 & 738.3 & 10 & 1130.7 \\
\hline 5 & 817.1 & 11 & 1183.4 \\
\hline 6 & 888.8 & 12 & 1233.9 \\
\hline
\end{tabular}

Whether, relations (31) and (32) are applicable for all the first generation baryons or applicable only for the ground state of the first generation baryon, is to be decided with further study.

\section{Conclusions}

Subject of final unification is having a long history. So far, no model succeeded in implementing the Newtonian gravitational constant or Planck scale in nuclear and electroweak interactions. Even though, the basic idea of String theory is very simple, very interesting and highly intuitive, there are no concrete new predictions on low energy scales and high energy scale predictions are beyond the reach of current technology. It is an indication of 'incompleteness' in String theory paradigm. In this paper, with reference to Planck scale, we presented a variety of relations pertaining to nuclear and electroweak coupling 
constants. It is clear from the above discussion that we could satisfactorily fit the nuclear data through empirical relations. This sincere attempt is to be ascertained by the scientific community. We would like to appeal that, with respect to currently believed String theory and Quantum gravity models - proposed assumption, proposed semi empirical relations, proposed procedure for understanding the hadronic mass spectrum, can be given some consideration in developing a 'workable model' of TOE.

\section{Acknowledgements}

Author Seshavatharam U.V.S is indebted to Professors shri M. Nagaphani Sarma, Chairman, shri K.V. Krishna Murthy, former chairman, Institute of Scientific Research in Vedas (I-SERVE), Hyderabad, India and Shri K.V.R.S. Murthy, former scientist IICT (CSIR), Govt. of India, Director, Research and Development, I-SERVE, for their valuable guidance and great support in developing this subject.

\section{REFERENCES}

[1] K. Becker, M. Becker and J. H. Schwarz. String Theory and M-theory: A Modern Introduction. Cambridge University Press, (2006).

[2] Edward Witten. What Every Physicist Should Know About String Theory. GR Centennial Celebration, Strings 2015, Bangalore, India. (2015).

[3] Juan M. Maldacena. Gravity, Particle Physics and Their Unification. Int.J.Mod.Phys. A15S1 840-852 (2000)

[4] Tilman Sauer. Einstein's Unified Field Theory Program. The Cambridge Companion to Einstein, M. Janssen, C. Lehner (eds), Cambridge University Press. 2013. (Chapter 9) http://philsci-archive.pitt.edu/3293/1/uft.pdf

[5] S. Bethke, G. Dissertori, and G.P. Salam. Quantum chromodynamics: Olive et al. (PDG), Chin. Phys. C38, 090001 (2014)

[6] C. J. Isham, A. Salam and J. Strathdee, 2+ Nonet as Gauge particles for SL(6, C) symmetry, Phys. Rev. D8, 8 (1973).

[7] Salam and J. Strathdee, Class of solutions for the strong-gravity equations, Phys. Rev. D16, 8 (1977).

[8] Sivaram and K. P. Sinha, Strong Spin-two Interaction and General Relativity, Phys. Rep. (Rev. Sect.Phys. Lett.) 51, 3 (1979).

[9] Dj. Sijacki and Y. Ne'eman, QCD as an effective strong gravity, Phys. Lett. B247, 4 (1990).

[10] Y. Ne'eman and Dj. Sijacki, Proof of pseudo-gravity as QCD approximation for the hadron IR region and J _ M2 Regge trajectories, Phys. Lett. B276 (1992).

[11] Abdus Salam. Strong Interactions, Gravitation and Cosmology. Publ. in: NATO Advanced Study Institute, Erice, June 16-July
6,1972

[12] Salam A, Sivaram C. Strong Gravity Approach to QCD and Confinement. Mod. Phys. Lett, A8(4), 321- 326. (1993)

[13] C. Sivaram et al. Gravity of Accelerations on Quantum Scales and its consequences. Hadronic Journal, Vol. 38, No. 3, 283 (2015)

[14] O. F. Akinto, Farida Tahir. Strong Gravity Approach to QCD and General Relativity. arXiv:1606.06963v3

[15] Roberto Onofrio. Proton radius puzzle and quantum gravity at the Fermi scale. EPL 104, 20002 (2013)

[16] U. V. S. Seshavatharam and S. Lakshminarayana Towards a workable model of final unification. International Journal of Mathematics and Physics 7, No 1, 117-130 (2016).

[17] U. V. S. Seshavatharam and S. Lakshminarayana. To Validate the Role of Electromagnetic and Strong Gravitational Constants via the Strong Elementary Charge. Universal Journal of Physics and Application 9(5): 216-225, 2015

[18] U. V. S. Seshavatharam, Lakshminarayana S. To confirm the existence of nuclear gravitational constant, Open Science Journal of Modern Physics. 2(5): 89-102 (2015)

[19] U. V. S. Seshavatharam, Lakshminarayana S. Final unification with Schwarzschild's Interaction. Journal of Applied Physical Science International 3(1): 12-22 (2015).

[20] U. V. S. Seshavatharam, Lakshminarayana S. Understanding Nuclear Stability, Binding Energy and Magic Numbers with Fermi Gas Model. Journal of Applied Physical Science International, 4 (2) pp. 51-59 (2015)

[21] Seshavatharam, U. V. S. and Lakshminarayana, S. The Possible Role of Newtonian, Strong \& Electromagnetic Gravitational Constants in Particle Physics. Prespacetime journal, Vol 7, Issue 5, pp. 857-888 (2016)

[22] U. V. S. Seshavatharam and S. Lakshminarayana. Final unification with three gravitational constants associated with nuclear, electromagnetic and gravitational interactions. International Journal of Advanced Astronomy. 4 (2) 105-109 (2016)

[23] U. V. S. Seshavatharam and S. Lakshminarayana. Strong Nuclear Gravitational Constant and the Origin of Nuclear Planck Scale. Progress in Physics. Vol -3, 31-38, July 2010.

[24] U. V. S. Seshavatharam and S. Lakshminarayana. Understanding the basics of final unification with three gravitational constants associated with nuclear, electromagnetic and gravitational interactions. 61st DAE-BRNS Symposium on Nuclear Physics. F40, 942

[25] U. V. S. Seshavatharam and S. Lakshminarayana Understanding nuclear stability and binding energy with nuclear and electromagnetic gravitational constants. 61st DAE-BRNS Symposium on Nuclear Physics. A136. 332

[26] U. V. S. Seshavatharam and S. Lakshminarayana. Understanding nuclear structure with Schwarzschild interaction and Avogadro number. Proceedings of the DAE-BRNS Symp. on Nucl. Phys. 60 (2015). F8, 852

[27] U.V. S. Seshavatharam and S. Lakshminarayana. Analytical estimation of the gravitational constant with atomic and nuclear physical constants. Proceedings of the DAE-BRNS Symp. on Nucl. Phys. 60 (2015) F7, 850 
[28] U. V. S. Seshavatharam and S. Lakshminarayana. On the role of strong interaction in understanding nuclear beta stability line and nuclear binding energy. Proceedings of the DAE-BRNS Symp. on Nucl. Phys. 60 (2015) A32, 118.

[29] U. V. S. Seshavatharam and S. Lakshminarayana .Super Symmetry in Strong and Weak interactions. Int. J. Mod. Phys. E, Vol. 19, No. 2, p. 263-280 (2010).

[30] U. V. S. Seshavatharam and S. Lakshminarayana. SUSY and strong nuclear gravity in (120-160) GeV mass range. Hadronic journal, Vol-34, No 3, (2011) June, p. 277-300.

[31] U. V. S. Seshavatharam and S. Lakshminarayana. Calculating the energy of electron in H-atom using modified SUSY physics. Journal of Nuclear Physics, Material Sciences, Radiation and Applications Vol. 2, No. 2 February 2015 pp. $135-147$.
[32] U. V. S. Seshavatharam and S. Lakshminarayana Integral charge SUSY in Strong nuclear gravity. Proceedings of the DAE Symp. on Nucl. Phys. 56, 842-843 (2011)

[33] U. V. S. Seshavatharam and S. Lakshminarayana. Nucleus in Strong nuclear gravity. Proceedings of the DAE Symp. on Nucl. Phys. 56 (2011) A57, 302.

[34] U. V. S. Seshavatharam and S. Lakshminarayana. Role of SUSY in finding the equivalent term for the first three terms of the semi empirical mass formula. Proceedings of the DAE Symp. on Nucl. Phys. 58 (2013) A118, 284.

[35] K.A. Olive et al. (Particle Data Group), Chin. Phys. C, 38, 090001 (2014)

[36] Hua-Xing Chen et al. A review of the open charm and open bottom mesons. arXiv:1609.08928v1 [hep-ph] 28 Sep 2016. 